\title{
Geometría avanzada para espacios de gestión ciudadana: un enfoque desde la Creatividad Colectiva
}

\author{
Advanced geometry for spaces of citizen management: \\ an approach from the Collective Creativity
}

\author{
Martín-Mariscal, Amanda \\ Universidad de Sevilla (España) \\ ammariscal@us.es \\ Martín-Pastor, Andrés \\ Universidad de Sevilla \\ archiamp@us.es
}

\author{
López-Martínez, Alicia \\ Universidad de Sevilla \\ alicia.Ipmt@gmail.com \\ Chiarella, Mauro \\ CONICET/Universidad Nacional del Litoral \\ chiarell@fadu.unl.edu.ar
}

\begin{abstract}
This work proposes the collective creativity processes for the creation of light architectures developed through advanced geometry. The aim is to provide contemporary solutions to citizen management areas. Nowadays, this type of urban areas is flourishing and seems to be interesting for parametric design and digital production to offer solutions to certain social needs via "High Tech" and "Low Cost" architectures created by collaborative processes.
\end{abstract}

Keywords: Creatividad Colectiva; Procesos Colaborativos; Geometría Avanzada; Arquitectura Ligera; Fabricación Digital; Diseño Paramétrico.

Keywords: Collective Creativity; Collaborative Processes; Advanced Geometry; Lightweight Architecture; Digital Fabrication; Parametric Design.

\section{Introducción}

En los últimos años han nacido multitud de iniciativas ciudadanas que hacen frente a complejos problemas de la ciudad contemporánea. En este contexto se han llevado a cabo proyectos con el objetivo de activar espacios urbanos obsoletos o en desuso mediante actividades de integración y regeneración urbana. Sin embargo el capital material necesario en los procesos arquitectónicos convencionales representa un obstáculo para el desarrollo de innovación en arquitectura aplicada a estas iniciativas. Junto a los escasos recursos económicos, se han generado unas arquitecturas e instalaciones autoconstruidas, casi a modo de emergencia, sin la planificación, los medios y el conocimiento necesarios para desarrollar una arquitectura acorde a las diferentes necesidades sociales.

El desarrollo de nuevos procesos de producción arquitectónica, basadas en procesos colaborativos y tecnologías digitales, ofrecen nuevas oportunidades para el desarrollo de arquitecturas ligeras, flexibles y de bajo impacto que puedan dar respuesta a algunas necesidades sociales de los espacios de gestión ciudadana. En este sentido, la accesibilidad a los laboratorios de fabricación digital, el desarrollo del conocimiento avanzado de todo tipo de superficies geométricas, el manejo de las herramientas CADCAM, el diseño paramétrico, y el aumento en la aplicación de experiencias de creatividad colectiva en las ciudades, brindan muchas opciones para mejorar los procesos de producción arquitectónica actuales.

\section{Metodología}

El método de trabajo se ha basado en las siguientes etapas: identificación de desajustes, determinación del espacio de acción, definición de las transformaciones y propuesta de solución. En primer lugar se ha identificado, dentro del ámbito de problemas complejos urbanos, la proliferación de espacios en desuso, los cuales están siendo reactivados mediantes iniciativas de gestión ciudadana pero muchas veces en modo de emergencia y desarrollando soluciones transitorias. En segundo lugar se establece como espacio de acción la posibilidad de mejorar los procesos de producción arquitectónica, estudiándose de forma integral desde la docencia universitaria y desde la práctica profesional. En tercer lugar se definen los espacios de transformación de los procesos, que en este caso se han centrado en el desarrollo de la geometría avanzada y de los procesos de creatividad colectiva. $Y$ finalmente se propone la implementación de arquitecturas colectivas ligeras como posible forma de mejorar la creación, diseño, fabricación e instalación de soportes para el desarrollo de actividades sociales en espacios urbanos en proceso de activación (Figura 1). 


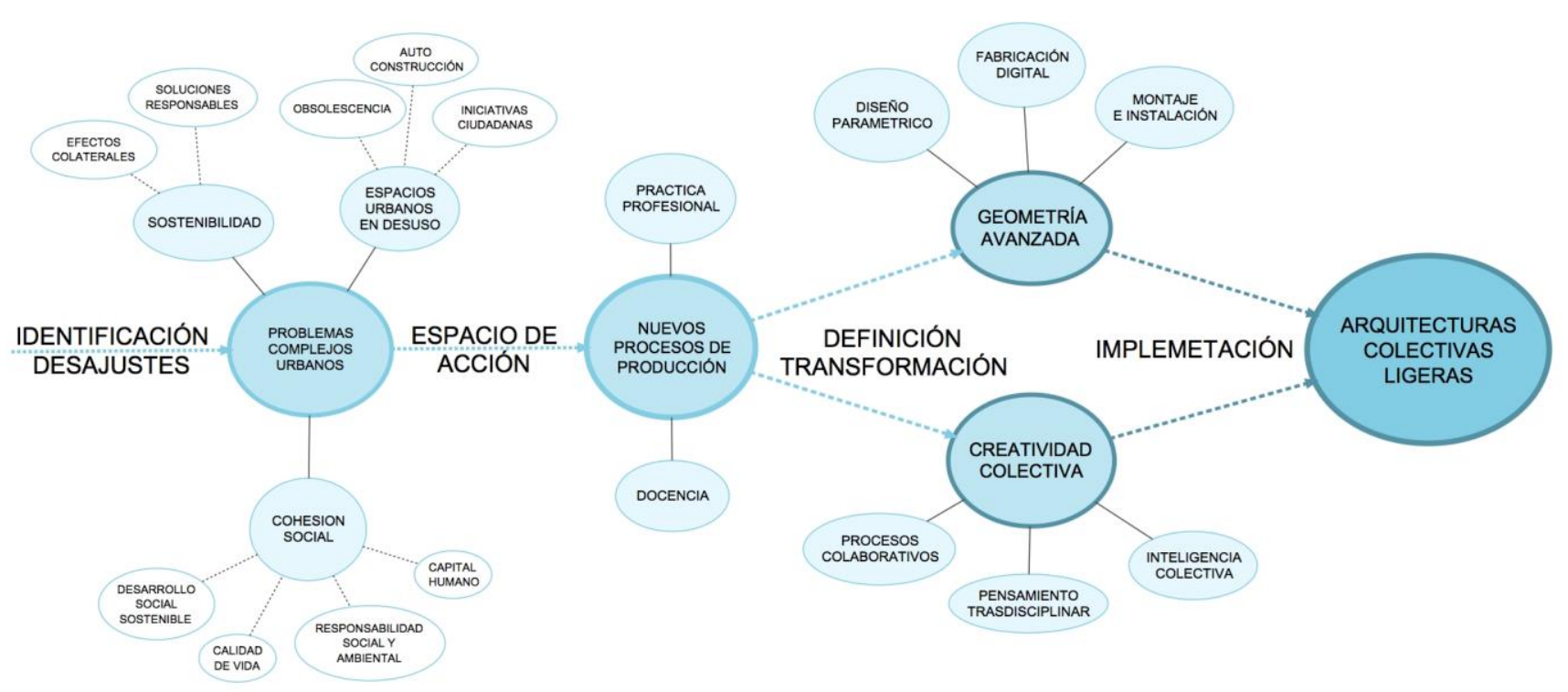

Figura 1: Metodología de ideación de Arquitecturas Colectivas Ligeras. Gráfico elaborado por Amanda Martín-Mariscal (2016)

En este sentido se han estudiado experiencias previas de construcción de elementos desarrollados para posibilitar las actividades sociales impulsadas en espacios urbanos gestionados por ciudadanos. Analizando si podrían mejorarse estos elementos mediante procesos de producción más avanzados y eficaces. Posteriormente se propone la integración de la geometría avanzada y los procesos colaborativos como proceso de producción arquitectónica y social en este tipo de espacios. Estos procesos pretenden dar una mejor respuesta a los requerimientos de estos espacios mediante arquitecturas colectivas ligeras.

\section{De la identificación de desajustes al espacio de acción}

\section{Problemas complejos urbanos}

"No se puede actuar racionalmente sin asumir la complejidad de la realidad". (Innerarity, 2008, p.94)

En contextos de crisis es cuando se hace más necesario realizar un trabajo de adaptación a los cambios, a través de la transformación de los procesos obsoletos. En este sentido, la reflexión profunda sobre la complejidad de los problemas urbanos actuales está desencadenando infinidad de iniciativas ciudadanas para la reinvención de los espacios públicos. El desafío de estas iniciativas es recuperar el derecho a la ciudad y hacer más humanos los lugares compartidos, de manera que avancemos más allá de las funciones de movilidad y de comercio, para fomentar las relaciones humanas y recuperar la cohesión social que debe caracterizar a nuestras ciudades. Crear espacios para el encuentro, para el arte y la cultura, para el aprendizaje,... no es incompatible con otras iniciativas ya implantadas en la ciudad. Se trata de enriquecer las relaciones sociales, que al final es una de las mayores oportunidades que ofrece vivir en espacios comunes con infraestructuras, tecnología y servicios disponibles para ello.
El desarrollo social sostenible como reto junto al deseo de mejorar la calidad de vida mediante la responsabilidad social y ambiental, son cuestiones ineludibles en el mundo contemporáneo. De forma que, aprovechando el capital humano de nuestras urbes, se están generando proyectos altamente interesantes y que desarrollan como espacios de responsabilidad: el cuidado y respeto por los ecosistemas, la búsqueda de la cohesión social, el impulso de la colaboración y la activación de la creatividad. Asimismo se está haciendo un intenso esfuerzo por ser conscientes de la complejidad del mundo en el que vivimos y por considerar los efectos colaterales de las acciones. Por tanto para resolver estos problemas complejos se hace necesaria la renovación de los procesos de producción desde una mayor complejidad y con una mirada poliédrica que considere todos los aspectos posibles desde a búsqueda del compromiso social.

\section{Nuevos procesos de producción arquitectónica}

«Existen interesantes oportunidades para los arquitectos, fundamentalmente para reinventar el proceso de diseño y fabricación, y para prever la integración de la tecnología en la vivienda de forma que responda a las necesidades y valores de sus habitantes y a los desafíos demográficos, de salud y energéticos de la sociedad. En el proceso de mejorar drásticamente la calidad del entorno construido, podemos incluso crear un papel más central e importante para el arquitecto de hoy». (Bernstein, 2011, p.113)

El escenario actual demanda un compromiso de la arquitectura y una renovada conexión con la sociedad, desarrollando más el interés por lo común y redefiniendo las condiciones productivas actuales. En este sentido, se está demostrando que es posible pasar de la construcción de la ciudad con fines especulativos, a la creación de barrios como espacio de convivencia (Naredo 2004). Donde los arquitectos estrella y la idea de ciudad marca serán sustituidos por una 
arquitectura que reinventa el oficio desde un nuevo concepto de artesanía (Cara, Ruiz 2011). El artesano contemporáneo debe idear la forma de compatibilizar todos los medios para que sea posible la colaboración entre diseñadores, fabricantes y otros profesionales. Así, la fabricación digital y el diseño paramétrico, aparecen como oportunidad para la integración de diseño y construcción. El arquitecto toma la forma del artesano, pero incorporando todas las herramientas disponibles, lo cual incluye computación, modelos experimentales y matemáticas (Martín-Mariscal, 2016). En esta línea nos interesa especialmente la siguiente definición de Richard Sennett: «Artesanía designa un impulso humano duradero y básico, el deseo de realizar bien una tarea, sin más» (Sennett, 2008, p.20).

En un mundo globalizado «la alquimia de la colaboración no surge de dos autores en una única voz, sino que prolifera para crear el coro de una multitud» (Hight, Perry 2006). En condiciones de extremada complejidad impera la colaboración por su capacidad de hacerse cargo de dicha complejidad e incluso de reducirla. En el escenario que nos ocupa, interesa que se active y desarrolle la creatividad, como impulsora de los procesos y catalizadora de un futuro, porque enfrentarse a la complejidad y a los desafíos es la forma en que, acertando o errando, caminamos hacia nuevos lugares más acordes con la contemporaneidad (Martín-Mariscal, 2016).

\section{Geometría Avanzada y Creatividad Colectiva para una renovación de la docencia en arquitectura}

«La profesión deberá sufrir una reconversión si quiere subsistir, empezando por los programas que ofrecen las universidades y la visión que se da a los alumnos del futuro de la profesión». (Landart, Gómez, de la Hoz, 2011:50)

Para que la adaptación de la arquitectura a las nuevas condiciones y desafíos tenga una repercusión en el futuro, es importante germinar cambios estratégicos y nuevos procesos en las aulas. Desde el aprendizaje se puede generar una mayor estabilidad en los cambios introducidos, y sobre todo se puede liberar una energía que se multiplique y propague en el futuro. La renovación metodológica tiene una evolución, muchas veces, demasiado lenta para la velocidad que demandan los cambios en la sociedad. Ante este escenario, la docencia puede colocar las bases que sustenten un futuro más avanzado para la arquitectura, donde los modelos de producción estén comprometidos con los problemas contemporáneos, y donde la arquitectura aproveche toda la potencialidad de la creación colectiva. De esta forma se estarán sentado las bases del futuro trabajo colaborativo, a través de los modelos de aprendizaje colectivo. Igualmente, la investigación también tiene la responsabilidad de trabajar intensamente sobre nuevos procesos en la arquitectura, y de explorar nuevas metodologías docentes basadas en la creatividad. Así que, cada vez más, necesitaremos que la docencia universitaria sea lo más flexible y ágil posible, para renovarse y para adaptarse con rapidez a los cambios y requerimientos de la sociedad (Martín-Mariscal, 2016).
Por un lado una sociedad en la que los colectivos están cada vez más articulados e interconectados mediante movimientos sociales y culturales, la creatividad colectiva se sitúa como el gran potencial de producción social, generando soluciones nuevas a los problemas que afectan profundamente a todos los estratos de las comunidades. Por otro lado, la renovación en la visión sobre la docencia de la geometría desde el pensamiento gráfico y mediante el uso de las herramientas digitales, también ofrece grandes potencialidades para renovar los procesos de producción arquitectónicos. En esta línea «el éxito de un proceso de aprendizaje creativo se debería reflejar no sólo en la capacidad para comprender la complejidad del fenómeno de la arquitectura en toda su dimensión para actuar adecuada y flexiblemente en el manejo de situaciones concretas, sino también en el descubrimiento de nuevos instrumentos conceptuales y operacionales que amplíen las posibilidades mismas del pensamiento proyectual» (Chiarella, Martín-Pastor,2015, p.2).

Las nuevas tecnologías CAD resuelven instrumentalmente las dificultades que podría suponer la representación, operando desde la propia geometría tridimensional y ofreciendo una amplia gama de recursos automatizados de control de la forma (Martín-Pastor, 2016:6). Gracias a una revisión profunda de la Geometría, con ayuda estas herramientas digitales, se inicia otra línea de trabajo fundamental para la renovación de los procesos de producción de la Arquitectura. Renovación que se ha comenzado a implementar en la docencia arquitectónica pero que también se está desarrollando en el ámbito profesional.

Ante esta propuesta de innovación de la docencia y la actividad profesional mediante la implementación de los procesos colaborativos y de los fundamentos de la geometría avanzada, a continuación se plantean propuestas para las necesidades detectadas en los espacios de gestión ciudadana. En los que la creatividad colectiva -entendida aquí como capacidad para resolver problemas de forma innovadora en el seno de un colectivo- y la aplicación avanzada de la geometría — «gramática que nos permite operar con razonamientos geométricos más elevados que finalmente sirven para articular problemas y soluciones concretas en arquitectura» (Martín-Pastor, 2016: 8)- se materializan en arquitecturas colectivas ligeras, sobre las cuales trataremos a continuación. 


\section{Arquitecturas colectivas ligeras en espacios de gestión ciudadana}

«Las ciudades están mutando de un lado a otro del globo. Los habitantes que antes sólo transitaban por sus calles 0 paseaban sus jardines se aposentan en ellas para amueblar plazas y ocupar solares. Una forma de urbanismo ciudadano emerge, a través del cual el espacio urbano es rediseñado en la calle y desde la calle» (Estalella, 2015)

Los espacios urbanos que nos ocupan en este estudio y que han proliferado en los últimos años, «evidencian que hay otra manera de tomar parte en la composición de la ciudad construyendo espacios y diseñando las infraestructuras necesarias para que podamos habitar en común» (Estalella, 2015). Existen muchos ejemplos de laboratorios urbanos actualmente, y se trata de «espacios urbanos autogestionados de todo tipo: espacios públicos, espacios sociales, espacios culturales, espacios centrales y periféricos, espacios ciudadanos, vecinales o como lo quieras llamar. Hay espacios espectaculares y recoletos, los hay de gestión híbrida, municipal o independiente, están ocupados, okupados, cedidos, alquilados... Espacios que han devuelto a la ciudad y a la calle su función imprescindible de escenario, entorno y ambiente de la vida en común» (Basurama, 2015)

Aunque se han producido numerosas experiencias a nivel internacional, nos hemos centrado en España ya que se ha constituido como un gran referente de la reivindicación del derecho a la ciudad y de la creación colaborativa. Así que -especialmente relacionado con el contexto de la crisis de 2008- se han desarrollado con éxito innumerables experiencias de este tipo, entre las cuales podemos destacar especialmente las siguientes por contar ya con una experiencia amplia:

\section{- Barcelona: el recinto fabril de Can Batlló, el Forat de la Vergonya y Recreant Cruïlles. \\ - Madrid: El Campo de Cebada, Esta es una plaza, \\ Tabacalera, Solarpiés, Solar Maravillas, Patio Maravillas y el Solar de Grilo (Figura 2). \\ - Badajoz: LaFábrikadetodalavida. \\ - Sevilla: el huero del Rey Moro, la Casa Grande del Pumarejo y Tramallol.}

En todos estos espacios se desarrollan actividades de todo tipo: huertos urbanos, teatro, cine, baile, deporte, cursos formativos... Todas orientadas para mejorar la calidad de vida en las ciudades, principalmente a través de la cohesión social y del cuidado del medioambiente. Se trata siempre de acciones creadas de forma altruista y con un alto nivel de austeridad, por eso nos parece especialmente interesante que las arquitecturas necesarias como soporte para su desarrollo respondan a estas condiciones. De forma que se trate de elementos de coste reducido, de fácil montaje y de bajo impacto, que han estado presentes en todos estos espacios desde sus inicios pero que han sido instalados provisionalmente y a modo de emergencia en muchos de los casos. Para ello será interesante hacer el uso más efectivo posible de la tecnología disponible, del conocimiento de la

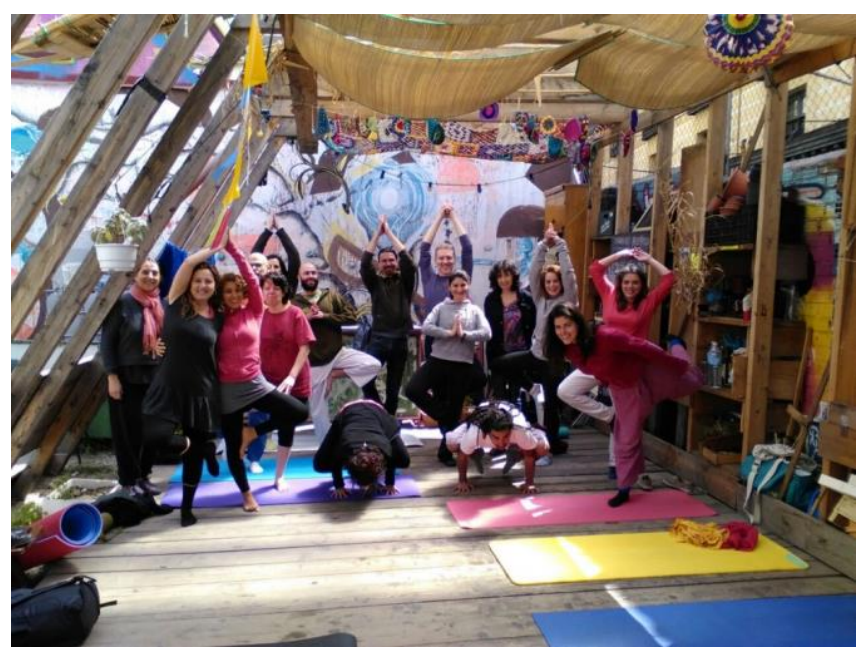

Figura 2: Espacio de gestión ciudadana de El Solar de Grilo.

geometría más avanzada y de la activación de las estrategias de creatividad colectiva. $Y$ en todo caso dando una gran importancia al valor social de estos espacios, tal y como explica el colectivo Paisaje Transversal con las siguientes palabras:

«La reformulación de los axiomas que han regido la arquitectura del siglo pasado, pasa en gran medida por subvertir la lógica objetual en lo procesual, reivindicando así el valor y potencial del proceso frente a la imagen, el icono. También resulta imprescindible recuperar el valor social de la práctica arquitectónica. Es decir, que seamos capaces de poner a disposición de la sociedad civil nuestros conocimientos y herramientas para favorecer transformaciones sociales». (Paisaje Transversal, 2013)

Las arquitecturas colectivas ligeras —basadas en procesos colaborativos y en la generación de formas basadas en la geometría avanzada- que se proponen en este trabajo son elementos que podrían dar respuesta a algunas necesidades de apoyo de las actividades llevadas acabo en los espacios definidos anteriormente. Se trata de pabellones (Figuras $4,5,6,7,8)$, desarrollados colaborativamente mediante procesos digitales de diseño paramétrico y tecnología de fabricación digital. El exhaustivo estudio geométrico permite crear formas que, de manera sencilla, pueden ser autoconstruidas por los ciudadanos con materiales económicos y a la vez ofrecer espacios útiles y de formas orgánicas. Las experiencias de diseño, fabricación y montaje de estos pabellones han demostrado buena viabilidad económica, gran adaptación de la forma y fácil montaje sin experiencia previa. A su vez se trata de un proceso global que resulta muy compatible con los procesos colaborativos que se producen en este tipo de espacios urbanos. 


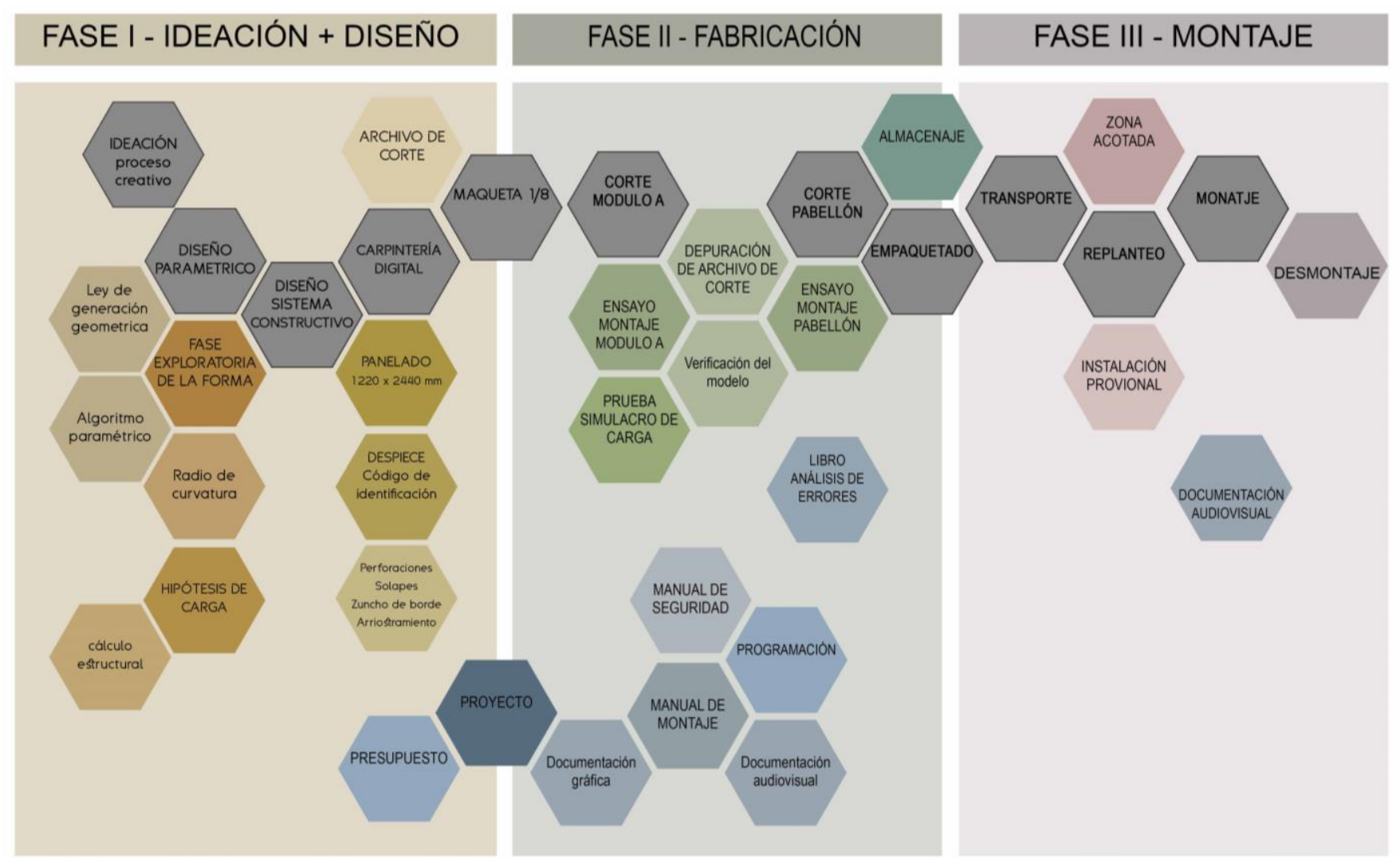

Figura 3: Fases de producción de los pabellones. Gráfico elaborado por Alicia López (2016)

\section{El proceso de producción de creatividad colectiva}

Las fases principales de producción de estos pabellones son las tres que se muestran en la imagen (Figura 3). Desde la primera fase de diseño es fundamental que se comience a trabajar de forma colaborativa, ya que todos los agentes implicados forman parte de una estructura horizontal en la cual se promueve una atmósfera de libertad, donde se necesita y se fomenta el apoyo mutuo. Se trata de trabajar siempre con equipos heterogéneos, lo cual enriquece todo el proceso a través de diversos puntos de vista, formas de trabajar y formación. También es importante fomentar la sinergia en toda la comunidad ya que a través de la retroalimentación continua se genera una interdependencia positiva que produce mejores resultados. Asimismo resulta interesante respetar la autoorganización de todos los agentes para conseguir una mayor fluidez y flexibilidad del proceso, de forma que ni los tiempos de actuación ni las tareas estén aislados. De esta manera, si se respetan los fundamentos de estos procesos colaborativos los efectos son más positivos, dándose las condiciones para que surja la creatividad colectiva.

\section{Diseño paramétrico y fabricación digital para una arquitectura colectiva ligera}

«Con las herramientas digitales hemos superado el problema de la representación de la tridimensionalidad en un soporte plano; así como el problema de la precisión en los trazados gráficos. También disponemos de una serie de automatismos a nuestro favor que aceleran el proceso de visualización y minimizan la energía mental usada en los procedimientos auxiliares intermedios, incorporando la potencia de visualización de la máquina, a nuestra mente ( $\mathrm{y}$ a nuestra imaginación), abriendo así nuevas relaciones asociativas.» (Martín-Pastor, 2016, p.8)

Los pabellones desarrollados hasta la actualidad se han creado mediante diseño paramétrico y fabricación digital, como ensayos de un estudio profundo para la aplicación de la geometría avanzada en la arquitectura. Partiendo de talleres de aprendizaje especializados, se han implementado procesos exhaustivos para exploración de formas innovadoras y su posterior construcción a escala 1:1. En esta etapa de la investigación se ha perseguido demostrar la importancia que tiene la geometría y su conocimiento avanzado para la arquitectura contemporánea, devolviendo a esta área de estudio el papel que merece para el desarrollo de la profesión. En todo momento ha sido fundamental comprender y manejar con un alto nivel las herramientas digitales del control del espacio, como instrumentos fundamentales para el pensamiento gráfico. Pero el auténtico objetivo es ir más allá del conocimiento de estas herramientas, para poder aplicar el conocimiento geométrico en el proceso de producción arquitectónico. 
El estudio de superficies desarrollables como investigación dentro del campo de la geometría avanzada permite la creación de pabellones de fácil montaje, ya que debido a la forma explorada pueden someterse a un proceso de fabricación digital. El trabajo de "carpintería digital" hace posible el despiece de las superficies planas, llevándola a la escala deseada, para ser cortadas por CNC. Una vez concluye el proceso de fabricación, el montaje parte desde el suelo, como superficie plana y desarrollable, donde en el espacio se curva en frío gracias a su forma. En el ámbito que nos ocupa en esta investigación posibilita multitud de maneras de responder a las necesidades de los espacios urbanos de gestión ciudadana. El proceso de producción unido al diseño de pabellones creados mediante superficies desarrollables ofrece una participación del usuario final. La aportación de un manual gráfico -incluso audiovisual- junto con talleres de formación sitúa al colectivo implicado en una situación de colaboración más enriquecida, implicándose en los trabajos donde su aportación consigue mayor calidad.

El optimizar el peso de los materiales junto con la búsqueda de materiales reciclados y reciclables así como el sistema de desmontaje y almacenaje, convierte a estos pabellones en arquitecturas sostenibles, de fácil reutilización o reciclado.

La experiencia previa de diseño, fabricación e instalación de cinco pabellones permite a partir de ahora avanzar desde el ensayo docente e investigador, a la aplicación en espacios reales con requerimientos funcionales y sociales precisos. Pasando del aprendizaje de los fundamentos de la geometría arquitectónica a la inclusión de los avances tecnológicos existentes para finalmente - aprovechando un indispensable entrenamiento previo- crear arquitecturas que cuenten con más libertad y las mínimas limitaciones, tanto en el diseño de la forma como en el aprovechamiento de la geometría para el abaratamiento de costes y la rapidez de instalación.

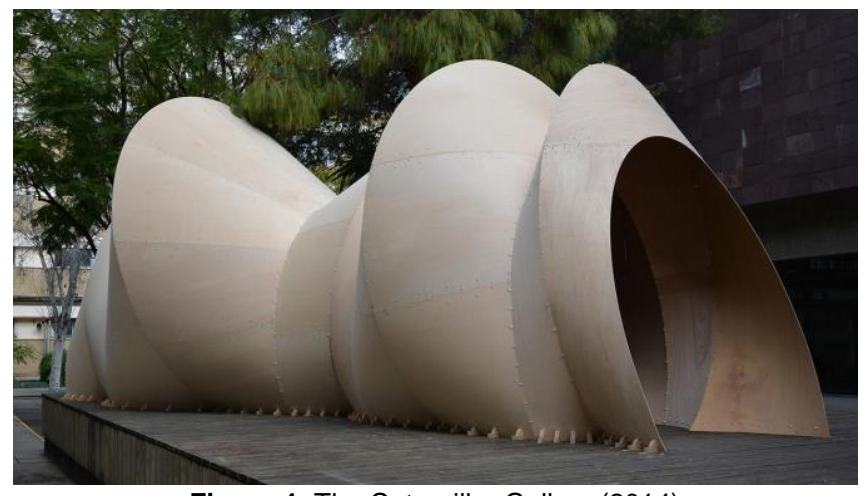

Figura 4: The Caterpillar Gallery (2014)

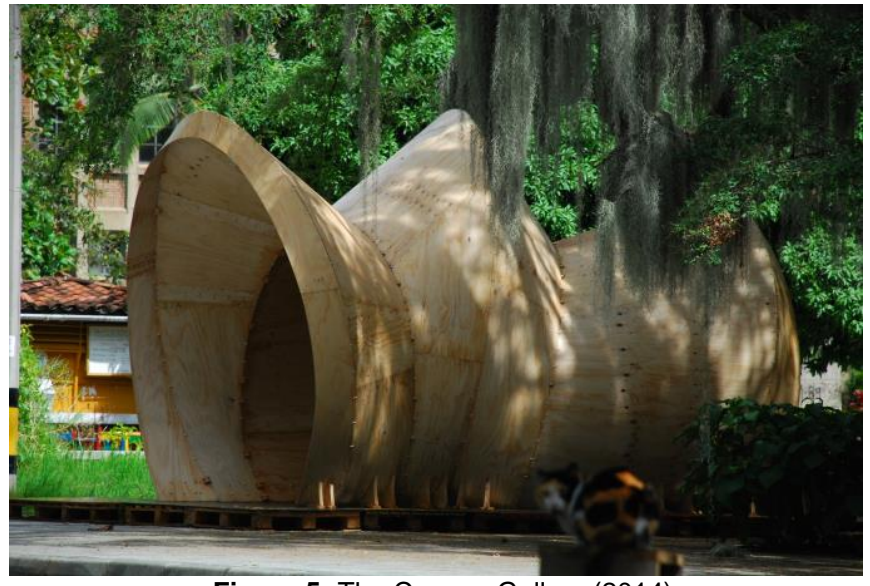

Figura 5: The Coocon Gallery (2014)

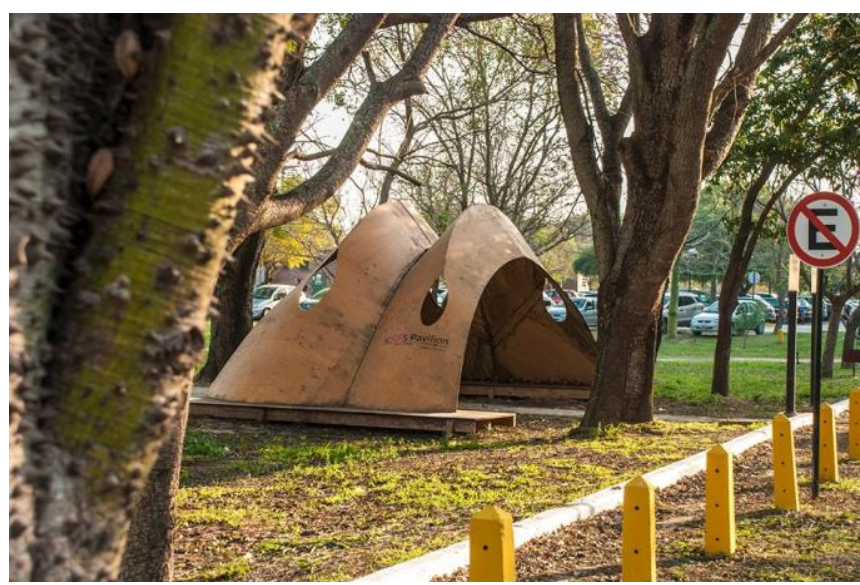

Figura 6: SFS Pavilion Santa - Fe (2015)

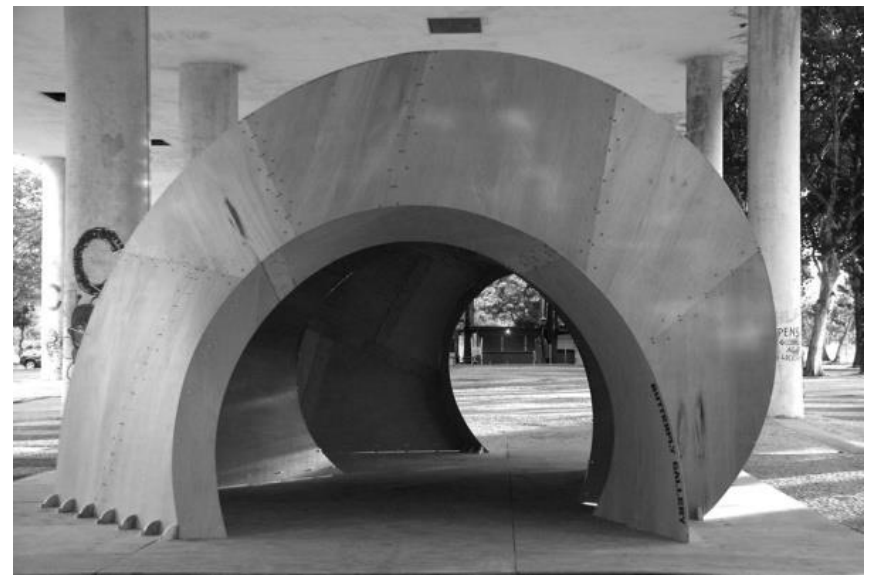

Figura 7: The Butterfly Gallery (2015) 


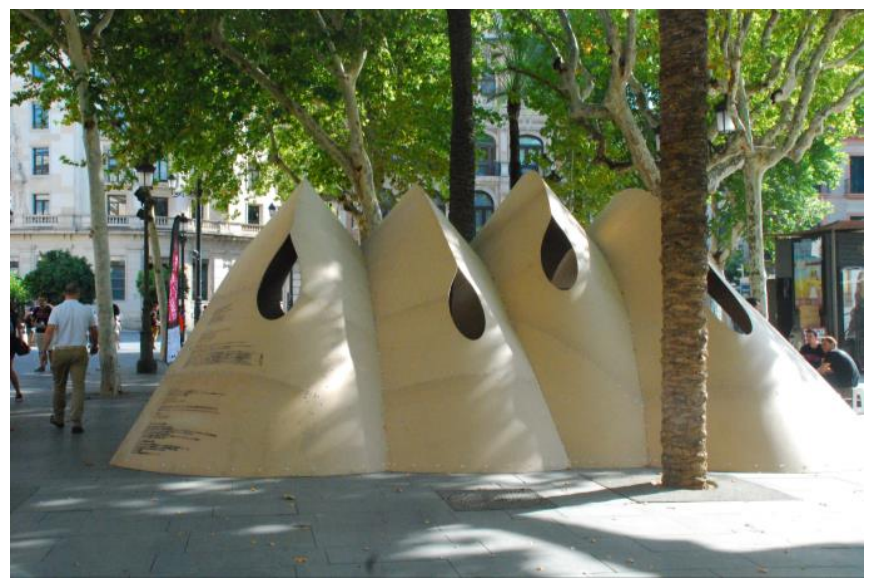

Figura 8: SSFS Pavilion Fab-Lab Sevilla (2015)

\section{Discusión}

Finalmente podemos exponer que la propuesta de aplicación de este tipo de arquitecturas ligeras, requiere por parte de los currículos universitarios un esfuerzo de renovación de las metodologías docentes. Ofreciendo un aprendizaje a los estudiantes de arquitectura e ingeniería que les ofrezca ampliar la perspectiva en el diseño y que fomente el estudio más profundo de posibilidades tanto formales como de respuesta a las necesidades sociales contemporáneas. En este sentido, y una vez adquirido este tipo de aprendizaje basado en la creatividad colectiva y en la geometría avanzada, se propone su exploración hacia el desarrollo de arquitecturas colectivas ligeras. Entendiendo que, ante las experiencias anteriores, este tipo de pabellones tienen cualidades muy interesantes que podrían ser beneficiosas en el apoyo a las actividades desarrolladas en los espacios de gestión ciudadana de nuestras ciudades.

Nos parece interesante el reto de adaptar este tipo de procesos de producción arquitectónico a los requerimientos de las actividades desarrolladas. Las instalaciones podrían añadir nuevas posibilidades a estos espacios comunes como lugares estimulantes y divertidos para niños- que más allá de cubrir necesidades de cobijo, a través de geometrías innovadoras, podrían constituirse como lugares especialmente sugerentes por las posibilidades de experiencia espacial que ofrecen. $Y$ al trabajar en espacios en los que habitualmente se producen procesos colaborativos, podría resultar bastante natural llegar a producir diseños en los que los usuarios pudieran participar de forma activa. La propia experiencia de incorporación al proceso de producción constituiría una actividad social en sí misma, así como la participación colectiva, de técnicos y ciudadanos, sería una experiencia innovadora e interesante para indagar.

En estos espacios, como ya se ha experimentado anteriormente por colectivos de arquitectos, hay infinitas posibilidades creativas de abrir múltiples posibilidades de disfrute del espacio urbano, incorporándose e integrándose en todos los procesos, administración pública, ciudadanos y técnicos. En esta línea el arquitecto actual avanza en el alcance de su rol, actuando muchas veces de mediador entre diferentes agentes o como posibilitador de la materialización de las ideas elaboradas colectivamente entre todos. De forma que estos agentes, los laboratorios de fabricación digital, las instituciones de todo tipo y el espacio urbano se unan con un único objetivo de mejorar la calidad en las ciudades a través de la cohesión social y el cuidado del medio ambiente. Siempre considerando los efectos colaterales con la finalidad de posibilitar un futuro mejor para todos.

\section{Agradecimientos}

\section{The Caterpillar Gallery}

Autores diseño y proyecto: Roberto Narváez-Rodríguez, Andrés Martín-Pastor, (En colaboración: Margarita Infante-

Pereda, María Aguilar-Alejandre)

Fabricación: equipo Fablab Sevilla

Montaje: Profesores y alumnos de las Escuelas ETSIE y

ETSA, Universidad de Sevilla.

Colaboración: Departamento de Ingeniería Gráfica

Universidad de Sevilla

\section{The Coccon Gallery}

Diseño y Proyecto: Andrés Martín-Pastor y Roberto NarváezRodríguez

Fabricación: Equipo Fablab Unal Medellín.

Ejecución y montaje: Estudiantes arquitectura UNAL Medellín y UNAL Manizales.

Coordinación: Escuela de Medios de Representación,

Facultad de Arquitectura. Unal Medellín Colombia.

Colaboración: Dto. Ingeniería Grafica, Equipo Fablab Sevilla.

\section{SSFS Pavilion - Santa Fe}

Diseño y Proyecto: Andrés Martín-Pastor, Roberto Narváez-

Rodríguez. Co-diseño paramétrico: Juan Expósito Bejarano.

Coordinación Académica y gestión: Mauro Chiarella.

Programa Maestría en Arquitectura, FADU, Universidad

Nacional del Litoral.

Fabricación y montaje: Alumnos y profesores Maestría en

Arquitectura, FADU.

Colaboración: Dto. Ingeniería Gráfica Universidad de Sevilla, ETSIE, Equipo Fablab Sevilla.

\section{The Butterfly Gallery}

Diseño y proyecto: Andrés Martín-Pastor,

Juan Expósito Bejarano.

Coordinación: María Angela.

Fabricación: LAMO3D, Foco Design

Montaje: colaborativo entre profesores y

alumnos de la UFRJ.

Colaboración: Dto. Ingeniería Gráfica Universidad de Sevilla, ETSIE, Equipo Fablab Sevilla.

\section{SSFS Pavilion - Fablab Sevilla}

Diseño y proyecto: Andrés Martín-Pastor, Juan Expósito

Bejarano y Roberto Narváez Rodríguez.

Fabricación: FABLAB Sevilla.

Jefe Producción: Daniel del Valle.

Montaje: colaborativo entre profesores y alumnos de la ETSIE, FABLAB Sevilla y Edificalab.

Colaboración: Dto. Ingeniería Gráfica Universidad de Sevilla, ETSIE, Equipo Fablab Sevilla. 
SIGraDi 2016, XX Congreso de la Sociedad Ibero-americana de Gráfica Digital

9-11, November, 2016 - Buenos Aires, Argentina

\section{Referencias}

Basurama. (2015). Manifiesto abierto por los espacios urbanos de madrid. Retrieved

http://www.laciudadviva.org/blogs/?p=27961

Bernstein, P., \& Deamer, P. (2010). Building (in) the future: Recasting labor in architecture. New Haven: Yale School of Architecture.

Chiarella, M., \& Martin-Pastor, A. (2015). Pensamiento gráfico y diseño colaborativo. Geometrías desarrollables en composiciones plegadas arquitectónicas. XIX congresso da sociedade iberoamericana de gráfica digital 2015 (pp. 702-707) Blucher Design Proceedings. Doi:10.5151/despro-sigradi2015-110107

Estalella, A. (2015). Colectivos de arquitectura: Otra sensibilidad urbana. Retrieved from http://www.prototyping.es/destacado/colectivos-de-arquitecturaotra-sensibilidad-urbana

Innerarity, D. (2008). Le futur et ses ennemis: De la confiscation de l'avenir à l'espérance politique [Los enemigos del futuro: una defensa de la esperanza política]. Paris: Flammarion-Climats.

Martín-Mariscal, A. (2016). Creatividad colectiva: Procesos de la arquitectura conteporánea en contextos de complejidad. (Unpublished).

Martín-Pastor, A. (2016). Un retorno a los fundamentos de geometría. The Butterfly Gallery - Helicoidal Surfaces, estrategias geométricas para la fabricación digital. Cadernos Proarq, 25, 130.

Paisaje Transversal. (2013). Friendly Madrid. El mapa ante el cambio de paradigma arquitectónico. Pasajes de Arquitectura y Crítica, (129), 39.

Rubio Landart, J., Gómez Perdomo, C., \& Hoz Castanys, R. d. I. (2011). Arquitectos en la encrucijada:¿qué puede hacer un arquitecto en la situación actual? Madrid: Alianza Editorial.

Sennett, R. (2008). The craftsman. New Haven: Yale University Press. 